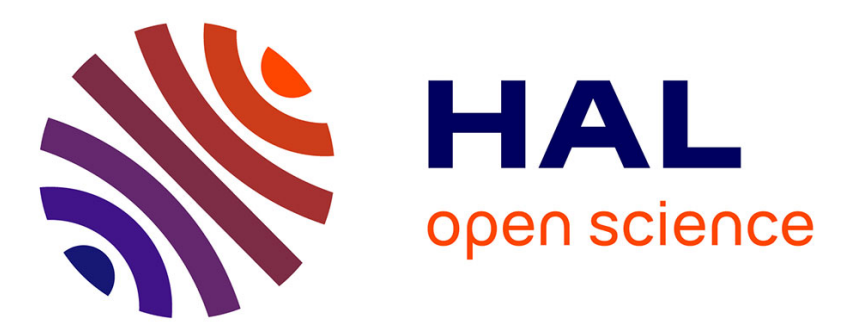

\title{
Effects of Stereo and Head Tracking on Distance Estimation, Presence, and Simulator Sickness using Wall Screen in Architectural Project Review
}

\author{
Sabah Boustila, Dominique Bechmann, Antonio Capobianco
}

\section{- To cite this version:}

Sabah Boustila, Dominique Bechmann, Antonio Capobianco. Effects of Stereo and Head Tracking on Distance Estimation, Presence, and Simulator Sickness using Wall Screen in Architectural Project Review. IEEE symposium on 3D User Interfaces (3DUI), 2017, Los Angeles, United States. hal03191206

\author{
HAL Id: hal-03191206 \\ https://hal.science/hal-03191206
}

Submitted on 6 Apr 2021

HAL is a multi-disciplinary open access archive for the deposit and dissemination of scientific research documents, whether they are published or not. The documents may come from teaching and research institutions in France or abroad, or from public or private research centers.
L'archive ouverte pluridisciplinaire HAL, est destinée au dépôt et à la diffusion de documents scientifiques de niveau recherche, publiés ou non, émanant des établissements d'enseignement et de recherche français ou étrangers, des laboratoires publics ou privés. 


\title{
Effects of Stereo and Head Tracking on Distance Estimation, Presence, and Simulator Sickness using Wall Screen in Architectural Project Review
}

\author{
Sabah Boustila* \\ Dominique Bechmann ${ }^{\dagger}$ \\ Antonio Capobianco
}

University of Strasbourg, France

\begin{abstract}
Stereo and head tracking are considered as distance perception cues in virtual environment. Several studies have investigated their influence on several tasks. Results were different among studies. In this paper, we conducted a complete experiment investigating the influence of the stereo and head tracking in the specific context of virtual visits of houses during architectural project review with clients. We manipulated the stereo and head tracking in four conditions and we examined effects of the two factors on distance perception (room dimensions, habitability, etc.), task difficulty, presence and simulator sickness. Results reveal a significant effect of the stereo on the estimation of the habitability, the dimensions of the rooms and task difficulty. However, the effect of stereo and head tracking was not significant on the presence and simulator sickness.
\end{abstract}

Index Terms: H.5.1 [Information Interfaces and Presentation]: Multimedia Information Systems-Artificial, augmented, and virtual realities; I.3.7 [Computer Graphics]: Three-Dimensional Graphics and Realism - Virtual reality

\section{INTRODUCTION AND RELATED WORK}

Visual human system provides several cues for the perception of distances such as binocular disparity, accommodation and convergence, etc. In virtual environments (VE), the visual stimulus is perceived through several display devices that can distort distance perception cues. Our work takes place in an architectural project aiming to perform virtual visits of houses with clients using virtual reality (VR) tools. During virtual visits, clients are supposed to judge the size of rooms and living comfort to validate (or not) the house' mockup. Thus, they need to evaluate distances.

Previous works have found that distances are misperceived in VEs [7, 2]. For more realistic perception, VR systems can provide head tracking, stereoscopy, etc. The stereoscopy has prompted many researchers to study its influence on distance estimation. Piryankova et al. [7] found that distances up to 2.5 are less underestimated with a stereoscopic display when using a large screen.

As for head tracking, Jones et al. [2] found similar underestimations in VE using HMD for distances between 2 and 8 meters whether using head tracking or not.

Moreover, these two advanced features are expected to increase the sense of presence, in comparison to desktop tools, since the perception is more similar to the real world when experiencing depth and point of view changes during body and head movements/shifting [1,5]. Indeed, in [1] participants reported a better sense of presence when seeing a rally racing on a monitor screen with the two factors. This is consistent with the result found in [5].

\footnotetext{
*e-mail: boustila@unistra.fr

†e-mail: bechmann@unistra.fr

$\ddagger$ e-mail: a.capobianco@unistra.fr
}

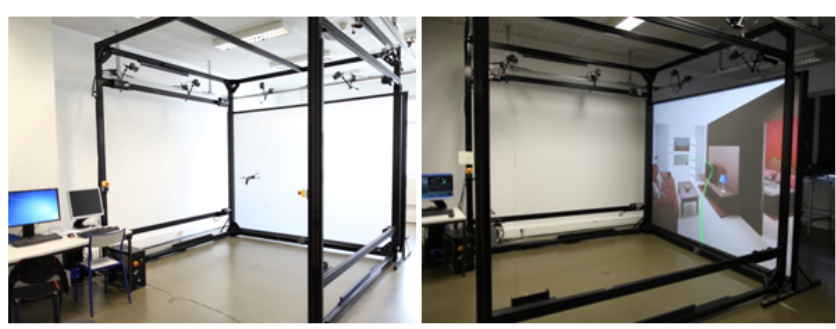

Figure 1: The platform In Virtuo. Right figure shows an example of our environment displayed on the screen.

Besides, most of previous works found that they affect the simulator sickness in VE [8, 4]. In facet, the score of simulator sickness was significantly higher when participants watched video game using stereo [8]. Same results were found by Keshavarz et al. [4].

In this paper, we conducted an experiment to investigate whether the advantages of the stereo and the head tracking affect the perception of distances, presence and simulator sickness in VE.

\section{EXPERIMENT DESIGN}

We varied the stereo and the head tracking by two levels. This provided a 2 X 2 design:

1) Stereoscopic vision + head Tracking (ST)

2) Stereoscopic vision + no head Tracking (SnoT)

3) Monoscopic vision + head Tracking (MT)

4) Monoscopic vision + no head Tracking(MnoT)

In the virtual visit, the participant walks virtually in the scene while standing physically at a distance of 1.5 meters in front of the screen. In the head tracking conditions, participants were encouraged to move their body and head inside the tracking space.

40 voluntary students in our university participated in the experiment. Ten individuals per condition.

\subsection{Apparatus and Stimuli}

We used a rear-projected wall screen $(3 \mathrm{~m} \times 2.25 \mathrm{~m})$ with a resolution of $1400 \times 1050$ and the Ninento Wiimote as interaction device. The platform is illustrated in figure 1.

Stereo was possible through active shutter glasses tracked using a Vicon tracking system based on 6 IR Bonita cameras $(200 \mathrm{~Hz})$ providing a tracked space of $6 \mathrm{~m}^{2}$. The refresh rate was of $60 \mathrm{~Hz}$ per eye. The virtual eyepoint height was set automatically corresponding to the subject's eye height. When the head tracking was not used, the experimenter calibrates this parameter before disabling the tracking system. Interocular distance was fixed to $6.3 \mathrm{~cm}$ for all participants.

Our program is developed in $\mathrm{C}++$ and is based on the VRJuggler library for device management. 


\subsection{Measurement}

We used a questionnaire to evaluate the perception of distances. 1) Distance estimation: closed-ended questions with 4 response choices (in meter), to evaluate the length and width of the rooms.

2) Size perception and habitability: 7-point Likert type scale questions, to evaluate specific possibility of furnishing and the comfort in the house, respectively.

3) Task difficulty: a 7-point Likert type scale question, to evaluate the difficulty of the virtual visit and distance estimation task.

Furthermore, we measured the presence (PQ) [10] and simulator sickness (SSQ) [3]. At the end, we performed a debriefing.

\subsection{Procedure}

The experiment consists of virtual visits of houses at scale 1:1. Before starting the experiment, participant reads instructions and trains on the navigation technique in the VE.

The experiment starts, participant moves using an active guided navigation following a predefined path and uses buttons on wiimote to move forward, to stop, and to turn around himself, rotations up and down and going back were not possible. When the participant is inside a room, he hears a beep and he gets stuck with only the ability to look around himself. The experimenter starts asking questions regarding distance perception. The participant answers orally. Afterwards, he unlocks the participant to visit the next room.

At the end, the participant answers the simulator sickness questionnaire, the presence questionnaire and gives a debriefing.

\section{Results and Discussion}

In the analysis, for each group of question we calculated the mean of signed gap between the participant's answers and the veridical answers. For the statistical analysis, we performed a nonparametric Kruskal-Wallis rank sum test at a 5\% significance level, and pairwise comparisons using the Nemeneyi post-hoc test.

Results revealed significant influence of stereo and head tracking on distance estimation ( $p$-value $=0.011)$ and habitability ( $p$-value $<0.05)$. Pairwise comparisons have shown significant differences between ST vs MT ( $p$-value $=0.026, p$-value $=0.001)$ and SnoT vs MT ( $p$-value $=0.026, p$-value $=0.015)$ for distance estimation and habitability respectively. We observe no significant difference between ST vs SnoT and MT vs MnoT. We confidently interpret this as an absence of effect for head tracking. Probably the amount of head motion was much smaller leading to no significant effects. Indeed, large screens provide display only on one screen in front of the view. Thus, even if participants were encouraged to move in the tracked space, they performed just small motions. Jones et al. [2] also found no significant effect of head tracking on distance estimations.

As for stereo, we found an effect on distance estimation and habitability only when the head tracking is used in ST and MT. Similarly, Willemsen et al. [9] have found no significant effect of stereo on distance estimation when head tracking were not used.

Concerning task difficulty we found significant result ( $p$-value $=0.016$ ). Task difficulty was estimated as being average with a mean values around 4/7 (7 represent a difficult task). Pairwise comparisons revealed a significant difference only between SnoT and MnoT ( $p$-value $=0.016)$. The task was more difficult when the two factors were removed (MnoT) with a mean of $4.3 / 7$ while easier with stereo (SnoT) with a mean of 3.4/7. This means that the head tracking alone do not influence task difficulty while the stereo do only when the head tracking is removed. The task difficulty assesses the difficulty of the experiment in the VE and the difficulty of the distance estimation task. Thus, for head tracking, the result seems logical because it did not affect the estimation tasks. As for stereo, previous work found that the importance of the stereo depends on the difficulty of the task to perform in VE [6].
Results revealed no significant effect of stereo and head tracking on size perception $(p$-value $=0.710)$ and presence $(p$-value $=$ $0.576)$. In contrast to previous work that found improvement in presence when using stereo and head tracking $[1,5]$, in our study, the presence was good in all conditions with means between 4.2 and 4.5 on 7 (7 is good). Probably, the influence of the two factors depends on the nature of the task. In our study, the participants visited houses and since the majority have already performed this task in real life, virtual visits seemed natural and probably led to a good sense of presence.

Furthermore, no significant effect of stereo and head tracking was found on simulator sickness $(p$-value $=0.144)$. This result is different from previous work that found significant influence of stereo in the context of videos and video games visualisation in VEs $[8,4]$.

\section{Conclusion}

In this paper, we investigated the effect of stereo and head tracking on the estimation of distances, the presence and simulator sickness during virtual visits of houses using a wall screen. To evaluate distance perception we used a specific questionnaire to evaluate wall dimensions (distance estimation), the possibility of specific furnishing (size perception) and the leaving comfort (habitability). Results revealed significant effect of stereo when used with head tracking only on distance estimation and habitability. Besides, task difficulty was higher when the two factors were removed. No significant influence was found on presence and simulator sickness.

In future work we will investigate other estimation methods such as direct walking. Furthermore, adapted IPD will investigated in an additional experimental condition.

\section{REFERENCES}

[1] W. A. IJsselsteijn, H. d. Ridder, J. Freeman, S. E. Avons, and D. Bouwhuis. Effects of stereoscopic presentation, image motion, and screen size on subjective and objective corroborative measures of presence. Presence, 10(3):298-311, June 2001.

[2] A. Jones, J. E. Swan, G. Singh, and E. Kolstad. The effects of virtual reality, augmented reality, and motion parallax on egocentric depth perception. In 2008 IEEE Virtual Reality Conference, pages 267-268, March 2008.

[3] R. S. Kennedy, J. M. Drexler, D. E. Compton, K. M. Stanney, S. Lanham, and D. L. Harm. Configural scoring of simulator sickness, cybersickness and space adaptation syndrome: Similarities and differences? Virtual and Adaptive Environments: Appplications, Implications, and Human Perfoormance, pages 247-278, 2003.

[4] B. Keshavarz and H. Hecht. Stereoscopic viewing enhances visually induced motion sickness but sound does not. Presence, 21(2):213228, May 2012.

[5] S. Lee and G. J. Kim. Effects of visual cues and sustained attention on spatial presence in virtual environments based on spatial and object distinction. Interacting with Computers, 20(45):491 - 502, 2008.

[6] R. H. Miller and R. J. Beaton. Some effects on depth-position and course-prediction judgments in 2-d and 3-d displays. Stereoscopic Displays and Applications II, 1991.

[7] I. V. Piryankova, S. de la Rosa, U. Kloos, H. H. Blthoff, and B. J. Mohler. Egocentric distance perception in large screen immersive displays. Displays, 34(2):153 - 164, 2013.

[8] J. Schild, J. LaViola, and M. Masuch. Understanding user experience in stereoscopic $3 \mathrm{~d}$ games. In Proceedings of the SIGCHI Conference on Human Factors in Computing Systems, CHI '12, pages 89-98, New York, NY, USA, 2012. ACM.

[9] P. Willemsen, A. A. Gooch, W. B. Thompson, and S. H. CreemRegehr. Effects of stereo viewing conditions on distance perception in virtual environments. Presence: Teleoper. Virtual Environ., 17(1):91101, Feb. 2008.

[10] B. G. Witmer and M. J. Singer. Measuring presence in virtual environments: A presence questionnaire. Presence: Teleoper. Virtual Environ., 7(3):225-240, 1998. 